\title{
Destabilization Control of a Chaotic Motor for Industrial Mixers
}

\author{
S. Ye and K.T. Chau \\ Department of Electrical and Electronic Engineering \\ The University of Hong Kong, Hong Kong, China \\ shuangye@eee.hku.hk,ktchau@eee.hku.hk
}

\begin{abstract}
Industrial mixers are among the most expensive and ineffective equipment in food, drug, chemical and semiconductor industries. Chaotic mixing has been proposed to improve the energy efficiency and the degree of homogeneity using either mechanical or electrical means. This paper presents the destabilization control of the DC motor (the agitator) for industrial mixing processes through the pole placement technique which is more analytically accessible compared with time-delay control proposed before. Theoretical derivation and computer simulation are provided to illustrate the controllable chaotic motion. Moreover, the mixing effectiveness is experimentally verified by an acid-base neutralization reaction.
\end{abstract}

\section{Keywords-chaotic; mixing; motors}

\section{INTRODUCTION}

Stirred tanks are one of the most important mixing devices in food, drug, chemical and semiconductor industries. They can be used for both continuous and batch processes, and can process single liquid phase as well as gas-liquid and solidliquid dispersions. One of the major limitations of stirred tanks is segregated regions formed in open impellers where turbulent mixing cannot be achieved [1]. The persistence of these segregated regions requires more energy consumption or higher rotational speed. As a result, industrial mixers are among the most expensive and ineffective equipment. The industrialists and academics in the USA have estimated that the cost of ineffective industrial mixing is of the order of US\$ 1 to 10 billion per annum [2]. Effects of this ineffectiveness are not only energy wastage [3], but also can be disastrous (a nuclearchemical waste explosion in Russia has been attributed to inadequate mixing of volatile compounds) [4]. While mixing can be improved by increasing the rate of stirring, such an approach is sometimes impractical. The product of many shearsensitive materials, such as proteins and other macromolecules, are damaged in high shear rates in fast stirring in biotechnological applications [1]. Thus, the improvement of mixing is highly desirable and justifiable.

In recent years, chaotic mixing has been proposed to improve the energy efficiency and the degree of homogeneity by using mechanical means [2] - [4]. These mechanical means are essentially based on the geometrically asymmetric design of the mixer to produce a practical chaotic motion. Instead of using mechanical means, our idea is to produce the desired chaotic motion electrically using destabilization control to improve mixing in a stirred tank.

Starting from the 1990's, a number of research activities on chaos in motors have been carried out. Most of them are based on the identification of chaos [5], the avoidance of chaos [6] and the stabilization of chaos [7] in various types of electric motors. Rather than negatively avoiding the occurrence of chaos in motors, the chaoization of the DC motor (the agitator) using time-delay feedback control is firstly proposed and implemented for use in industrial mixers [8]. Compared with those mechanical means, the electrically implemented chaoticmotion motor not only produces the desired chaotic mixing, but also offers the advantages of high flexibility and high controllability. The mixing performance of the controllable chaotic motor is encouraging compared with its constant speed counterpart.

The promising performance of the time-delay controlled mixer asks for more effort on the chaoization strategy to fully utilize the versatility of electrically implemented chaotic motion. Unfortunately, the effort on chaoization is rather less than it needs to be compared with the voluminous references on control of chaos, as chaos was once regarded as "unstable" and thus should be eliminated. Among the papers on chaoization, there are more successful methods of chaoization of discrete systems compared with continuous systems, which is more difficult. Unlike those chaoization methods used in continuous systems which require that the original system to have external oscillating excitation or periodic solution, timedelay feedback control enjoys the advantages of easy implementation and little requirement on the original system. The difficulty lies in the fact that delay differential equation hinders much of the analytical effort and allows mostly only the numerical analysis. However, the destabilization control [9] implemented through the pole assignment technique is more analytically accessible and is adopted in this paper.

There are many methods to evaluate the mixing processes, which can be divided into two categories: intrusive and nonintrusive. The intrusive methods include a probe or tracer put in the stirred tank to measure flow velocities and perturb the flow patterns that the investigators intend to measure. The nonintrusive methods, such as the Laser Doppler anemometer [10] and the acid-base neutralization reaction [11] are more attractive since they will not disturb the flow patterns.

This work was supported and funded by a grant (under project no. HKU7154/04E) from Research Grants Council of Hong Kong Special Administrative Region, China. 
Increasingly, the acid-base neutralization reaction takes the advantages of simple arrangement and low cost.

In Section II, theoretical derivation will be provided, and in Section III computer simulation will be presented to illustrate the proposed chaotic-motion motor. Then, the implementation of the mixing system will be introduced in Section IV. In Section $\mathrm{V}$, the results of chaotic mixing and constant speed mixing at the same power level will be compared to verify the effectiveness of the proposed chaotic mixing. Finally, conclusions will be drawn in Section VI.

\section{CHAOTIC-MOTION MOTOR}

The core of destabilization control is to destabilize a stable equilibrium point by linear feedback with unstable pole placement [9]. For an n-dimensional autonomous system:

$$
d \mathbf{x} / d t=\mathbf{f}(\mathbf{x}, \lambda)
$$

where $\mathbf{x} \in \mathbf{R}^{n}$ is the state vector, $\lambda \in R$ is a parameter. Assume that $\mathbf{f}$ is sufficiently differentiable for all variables and parameters and that the system has only one stable equilibrium point. The state vector and the parameter will then be described as:

$$
\begin{aligned}
& \mathbf{x}=\mathbf{x}^{*}+\xi \\
& \lambda=\lambda^{*}+u
\end{aligned}
$$

where $\mathbf{x}^{*}$ is the equilibrium and $\lambda^{*}$ is the nominal parameter. Then the variational equation can be written as:

$$
d \xi / d t=\mathbf{A} \xi+\mathbf{B} u
$$

where

$$
\begin{aligned}
& \mathbf{A}=\left.\frac{\partial \mathbf{f}}{\partial \mathbf{x}}\right|_{\mathbf{x}=\mathbf{x}^{*}, \lambda=\lambda^{*}} \\
& \mathbf{B}=\left.\frac{\partial \mathbf{f}}{\partial \lambda}\right|_{\mathbf{x}=\mathbf{x}^{*}, \lambda=\lambda^{*}}
\end{aligned}
$$

The state feedback can be constructed as:

$$
u=\mathbf{C}^{T} \boldsymbol{\xi}=\mathbf{C}^{T}\left(\mathbf{x}-\mathbf{x}^{*}\right)
$$

where $\mathbf{C}$ is a control vector and its superscript $T$ stands for transposition. Thus the original system becomes:

$$
d \mathbf{x} / d t=f\left(\mathbf{x}, \lambda^{*}+\mathbf{C}^{T} \xi\right)
$$

The characteristic equation of the controlled system is:

$$
\left|\mathbf{A}+\mathbf{B C}^{T}-\mu \mathbf{I}\right|=0
$$

where $\mathbf{I}$ is an n-dimensional identity matrix. In case that the system is completely state controllable, namely

$$
\operatorname{rank}\left[\mathbf{B}|\mathbf{A B}| \mathbf{A}^{2} \mathbf{B}|\ldots| \mathbf{A}^{n-1} \mathbf{B}\right]=n
$$

poles of the controlled system may be placed at any desired locations by means of state feedback through an appropriate state feedback gain matrix. Control is triggered whenever the orbit enters $\delta$, namely

$$
\left\|\mathbf{x}-\mathbf{x}^{*}\right\|<\delta
$$

and will last for time $\tau$. When the control is turned on, the poles of the system are placed in the right-half $s$ plane which corresponds to the expanding effect; while after time $\tau$, the poles of the system go back to the left-half $s$ plane which corresponds to the extracting effect. With appropriate combination of the control parameters, we are able to produce a positive average exponential rate of separation of two nearby initial conditions. In another word, we are able to get a chaotic attractor this way.

A permanent magnet DC motor is used as the agitator, which can be modeled as:

$$
\frac{d}{d t}\left(\begin{array}{c}
\omega(t) \\
i(t)
\end{array}\right)=\left(\begin{array}{cc}
-\frac{B_{m}}{J} & \frac{K_{T}}{J} \\
-\frac{K_{E}}{L} & -\frac{R}{L}
\end{array}\right)\left(\begin{array}{c}
\omega(t) \\
i(t)
\end{array}\right)+\left(\begin{array}{c}
-\frac{T_{l}}{J} \\
\frac{V_{i n}}{L}
\end{array}\right)
$$

where $B_{m}$ is the viscous damping coefficient, $J$ is the load inertia, $K_{E}$ is the back-EMF constant, $K_{T}$ is the torque constant, $L$ is the armature inductance, $R$ is the armature resistance, $T_{l}$ is the load torque, $V_{\text {in }}$ is the DC supply voltage, $\omega$ is the motor speed and $i$ is the armature current. In this paper, $\left\|\omega-\omega^{*}\right\|<\delta$ is adopted to get closer control of the range of motor speed, which is crucial to the mixing effect. However, this is not the only way that $\delta$ can be designed; complicated control which also takes current into triggering consideration is also applicable. Fig. 1 shows the control diagram.

The motion of the motor can be understood plotting armature current with respect to motor speed, which is called a phase portrait. The attractor takes a ring-like shape. The "center" of the attractor is determined by the equilibrium point. Its inner "radium" is decided by $\delta$, while the outer "radium" is determined by the combination of $\mathbf{C}$ and $\tau$. The destabilization control is implemented through the adjustment of $V_{i n}$. 


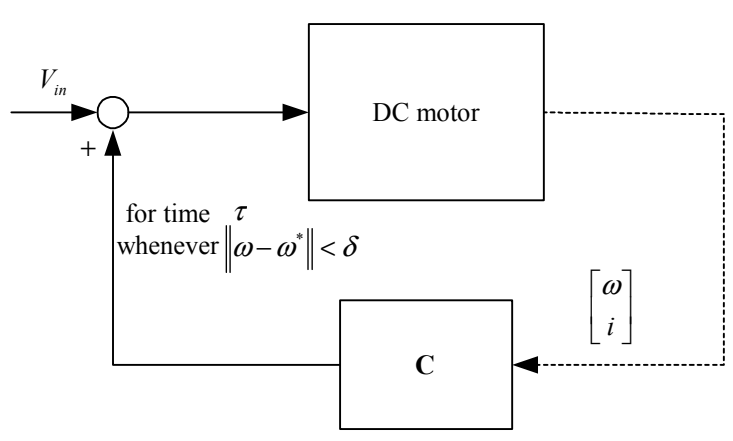

Figure 1. Control system diagram.

Unlike time-delay method which achieves chaos through the control of armature current, the pole assignment technique repels the orbit from the equilibrium via the control of armature voltage. The discussion above has the precondition that $V_{i n}$ is affordable. Practically, it isn't always the case. If the absolute value of the calculated $V_{i n}$ exceeds the rated voltage of the motor, the real supply voltage will be limited to be either a positive or a negative rated value. $V_{\text {in }}$ may saturate in case that the stretching of the space, $\tau$, or $\delta$ is large, or the combination of all these cases. It should be noted that the motion does not conform to the desired destabilization motion when saturation happens.

Chaotic motion produced through destabilization seems regular. Further work needs to be done on the more sophisticated control strategy, namely the combination of different $\mathbf{C}, \delta$ and $\tau$ which will make the motion more randomlike and add more harmonic components into the power spectrum. Furthermore, the saturation effect of $V_{\text {in }}$ should also be considered in theoretical analysis to fully describe the proposed motion.

\section{Simulation Results}

In order to conduct computer simulation, realistic system parameters are adopted. Table I summarizes some practical data of the DC motor. To illustrate the effect of all the control parameters on the destabilization control, waveforms of motor speed, armature current, supply voltage and the phase portrait are shown respectively.

Fig. 2 is obtained selecting $\mathbf{C}=\left[\begin{array}{ll}-10 & 8\end{array}\right]^{T}, \delta=5 \mathrm{rpm}, \tau=$ $0.004 \mathrm{~s}, T_{l}=0 \mathrm{Nm}$ and $V_{i n}=0 \mathrm{~V}$. The selection of $T_{l}$ and $V_{i n}$ locates the equilibrium at the zero point. As can be seen from the waveforms of both motor speed and armature current, stable and unstable motion mode presides alternatively. Fig. 2(d) presents the attractor with the equilibrium marked by a star $(*)$. It should be noted that the equilibrium is marked in all the other phase portraits the same way. The parameter settings for Fig. 2 assure that $V_{\text {in }}$ varies within the rated value; therefore there is no saturation effect in $V_{i n}$.

Selecting $T_{l}=0.001 \mathrm{Nm}$ and $V_{i n}=10 \mathrm{~V}$ and keeping all the other control parameters intact, the equilibrium is shifted to a non-zero point. Fig. 3 shows that not only the equilibrium, but all the corresponding waveforms and the phase portrait are shifted compared with Fig. 2.

Both Fig. 4 and Fig. 5 are produced with the same equilibrium as Fig. 3 but with different $\tau$ and $\delta$, respectively. For Fig. $4, \delta=5 \mathrm{rpm}, \tau=0.008 \mathrm{~s}$. As can be seen from Fig. 4 , a long $\tau$ leads to the saturation of $V_{i n}(24 \mathrm{~V})$, and this in turn causes the saturation of motor speed as well as armature current. For Fig. $5, \delta=12 \mathrm{rpm}, \tau=0.004 \mathrm{~s}$. Caused by a large $\delta$, Fig. 5 also shows the saturation effect in the waveforms of motor speed, armature current, and supply voltage. Compared with Fig. 3(d), Fig. 4(d) shows a thick "ring" because of the long $\tau$; Fig. 5(d) shows a thin "ring" due to the large $\delta$.

\section{TABLE I. MOTOR PARAMETERS}

\begin{tabular}{ll}
\hline$B$ & $0.0002 \mathrm{Nm} /(\mathrm{rad} / \mathrm{s})$ \\
$J$ & $0.0000010388 \mathrm{Nm} /\left(\mathrm{rad} / \mathrm{s}^{2}\right)$ \\
$K_{E}$ & $0.04975 \mathrm{~V} /(\mathrm{rad} / \mathrm{s})$ \\
$K_{T}$ & $0.04998 \mathrm{Nm} / \mathrm{A}$ \\
$L$ & $1.5 \mathrm{mH}$ \\
$R$ & $7 \Omega$ \\
$V_{\text {rated }}$ & $24 \mathrm{~V}$ \\
\hline
\end{tabular}

\section{IMPLEMENTATION}

The mixing apparatus consists of a tank (a one liter glass beaker) and an impeller spun by a digitally-controlled drive (a SANYO R406 DC servo motor) mounted vertically on a stand (a BOSCH BS 35 drill holder) with its shaft positioned at the center of the tank. The shaft is mounted through a holding plate, which ensures consistent positioning between experiments and minimizes oscillations of the shaft tip. The whole set-up is shown in Fig. 6.

It should be noted that the mixture with low or moderate Reynolds number are particularly difficult to achieve effective mixing since the corresponding flow is laminar, whereas the mixture with high Reynolds number can easily achieve effective or so-called turbulent mixing. Thus, in this paper, a viscous mixture (light corn syrup) is purposely adopted so that the mixing effectiveness can be evaluated.

Firstly, the tank is filled with $200 \mathrm{ml}$ light corn syrup, $5 \mathrm{ml}$ $\mathrm{pH}$ indicator solution (universal indicator) and $5 \mathrm{ml}$ solution of $1 \mathrm{~N} \mathrm{HCl}$. The system is mixed until a uniform red color is observed since the solution is acidic. Next, a solution of well mixed solution (in dark green) of $100 \mathrm{ml}$ light corn syrup, 2.5 $\mathrm{ml} \mathrm{pH}$ indicator solution and $2.5 \mathrm{ml} 1 \mathrm{~N} \mathrm{NaOH}$ is added into the tank. Although the solution is acidic as there is twice as much acid as base, there are dark green regions due to diffusion limitations caused by the highly viscous solvent.

The beginning of the mixing of the acid-base solution is set as the zero of the time axis. The mixer is controlled by the dSPACE digital controller to perform chaotic mixing or normal constant speed mixing. The experiments are recorded using a web camera focused at the impeller. 


\section{EXPERIMENTAL RESULTS}

To assess whether chaotic mixing is more effective than normal constant speed mixing, it is equivalent to evaluate whether chaotic mixing can offer a more homogenous mixture under the same amount of energy consumption, or to evaluate whether chaotic mixing consumes less energy if homogeneity is achieved in both cases. The experiment is designed to compare the mixing time needed to achieve homogeneity using the same average power. First, the chaotic mixing experiment is conducted. The armature voltage and current are measured and the power is integrated to calculate the energy consumed to achieve homogeneity. Then, constant speed mixing is conducted at the same average power.

Fig. 7 shows the system coloration during chaotic mixing. Since twice as much acid as base is used in the experiment, the mixed regions of the stirred tank contain excessive acid, causing the indicator in these regions to appear red. On the other hand, the unreacted regions contain base and display a green color. Fig. 7(a) is taken after 9 s of mixing. Fig. 7(b) and Fig. 7(c) show the system coloration after $14 \mathrm{~s}$ and $20 \mathrm{~s}$ of mixing, respectively. The green color is less and less. After 60 $\mathrm{s}$, it reaches a uniform red color which is shown in Fig. 7(d).

The corresponding chaotic speed is bounded below 1000 rpm. Although instantaneous peek power reaches about $20 \mathrm{~W}$, the average power consumed is found to be only $1.4 \mathrm{~W}$ due to the bidirectional armature current which offsets part of the instantaneous power and reduces the average power level as a result.

Fig. 8 shows the results of constant speed mixing which adopts the speed of $350 \mathrm{rpm}$ at the same power level. In Fig. $8(\mathrm{a})$, the system has been stirred for $9 \mathrm{~s}$. It can be seen that the dark green region resides above the impeller. The dark green fades after $14 \mathrm{~s}$ of mixing as shown in Fig. 8(b). After $20 \mathrm{~s}$ of mixing, a segregated region above the impeller presents itself with smeared boundary as shown in Fig. 8(c). Fig. 8(d) shows a decreased size of the segregated region as well as a clear boundary between the segregated region and the rest of the mixture after $300 \mathrm{~s}$ of mixing.

Our experiments demonstrate that a large amount of energy needs to be consumed to destroy the segregated region, which hinders efficient mixing in the constant speed case. They also show that chaotic mixing effectively prevents the formation of such segregated regions, leading to effective and fast mixing.

\section{CONCLUSIONS}

In this paper, chaotic mixing is electrically implemented using destabilization method on a DC motor, which acts as the agitator. Experimental results demonstrate that chaotic mixing prevents the formation of segregated regions, thus leading to efficient mixing compared with normal constant speed mixing.

Further work needs to be done on the theory of destabilization. Firstly, to make the motion more random-like, a more sophisticated control strategy which combines different control parameters should be studied. Secondly, the saturation effect of supply voltage should be considered.

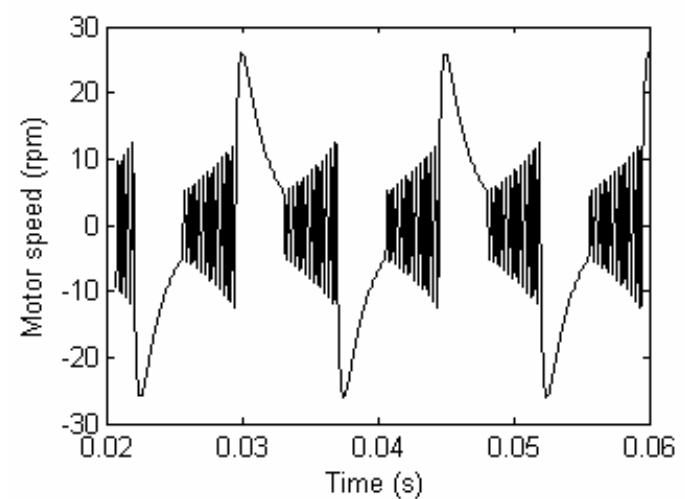

(a)

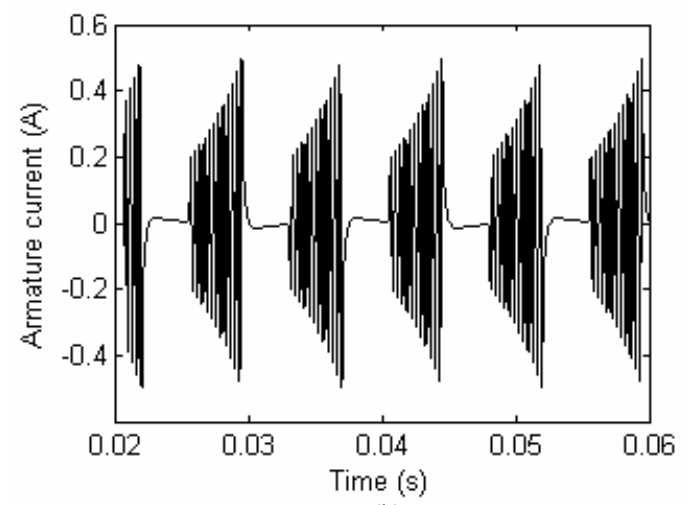

(b)

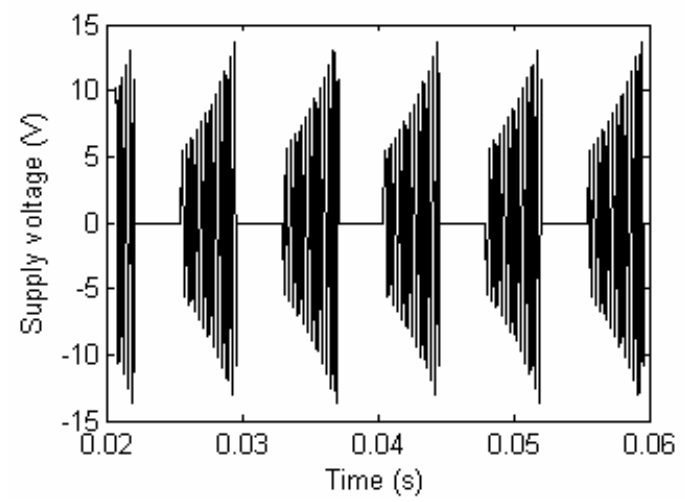

(c)

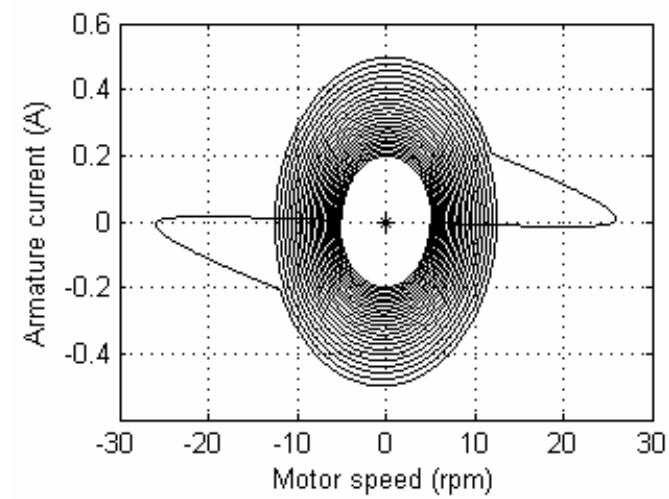

(d)

Figure 2. The equilibrium locates at the zero point. (a) Motor speed. (b) Armature current. (c) Supply voltage. (d) Phase portrait. 


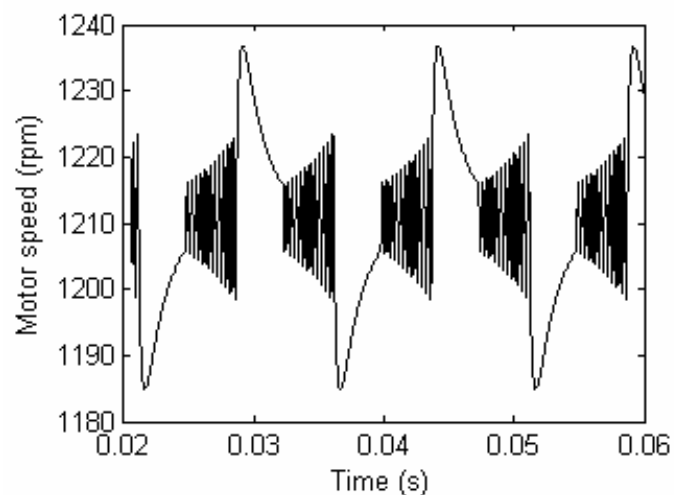

(a)

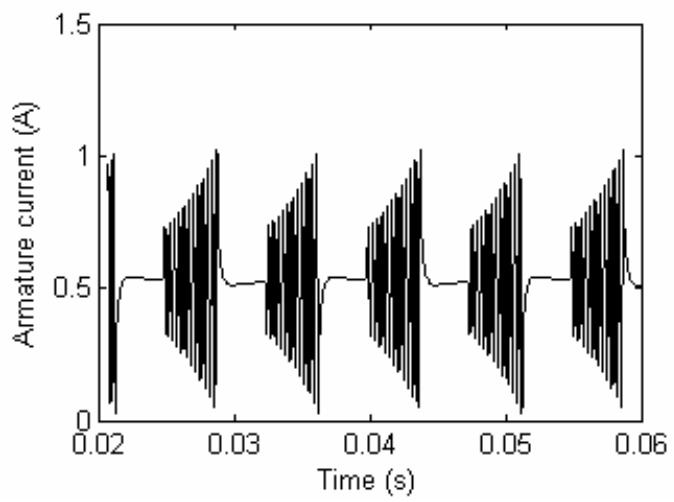

(b)

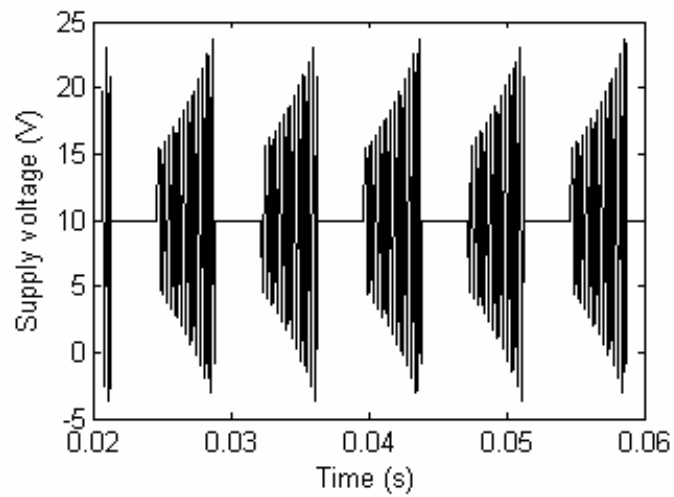

(c)

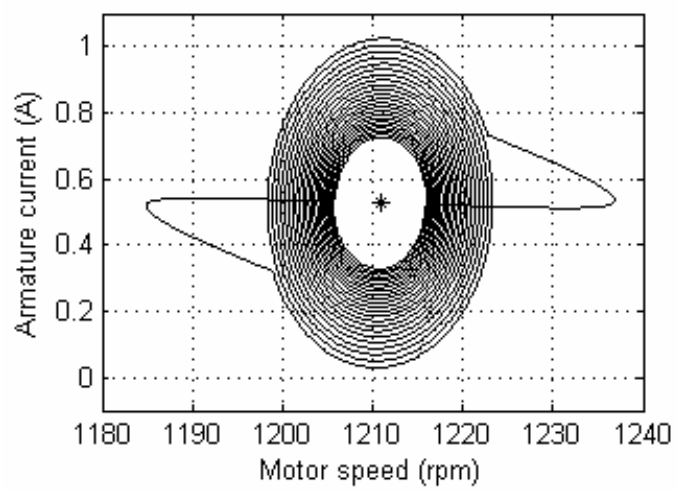

(d)

Figure 3. The equilibrium locates at a non-zero point. (a) Motor speed. (b) Armature current. (c) Supply voltage. (d) Phase portrait.

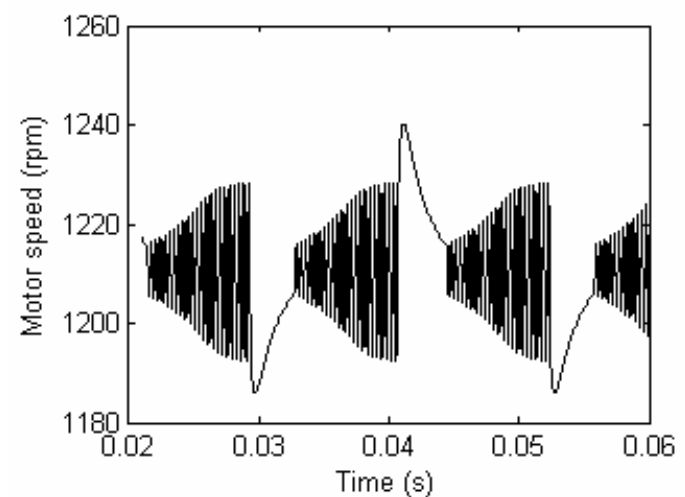

(a)

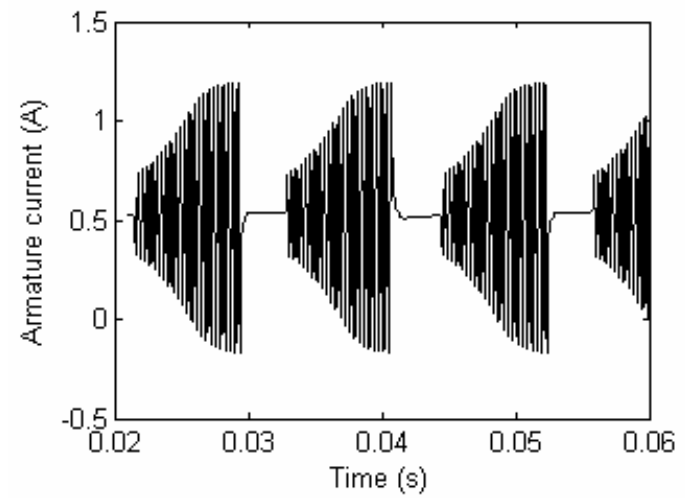

(b)

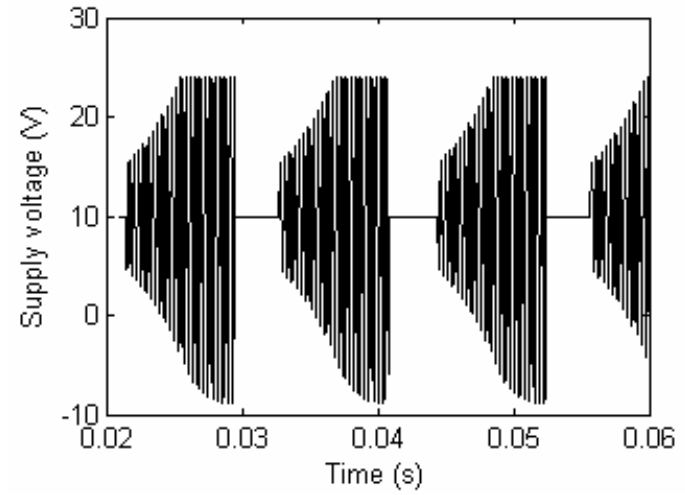

(c)

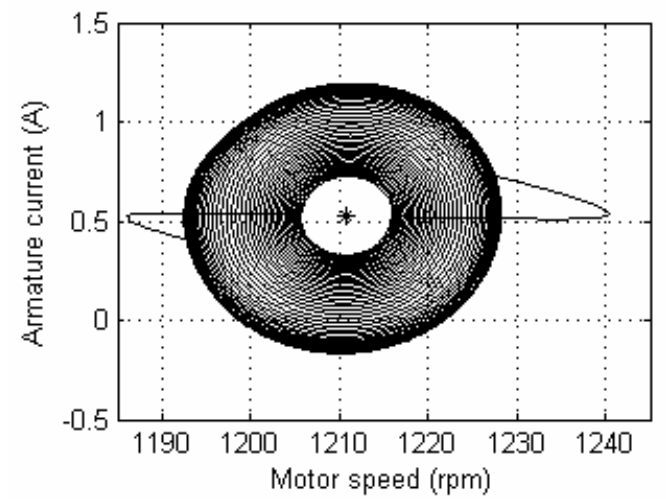

(d)

Figure 4. The equilibrium locates at a non-zero point with a long $\tau$. (a) Motor speed. (b) Armature current. (c) Supply voltage. (d) Phase portrait. 


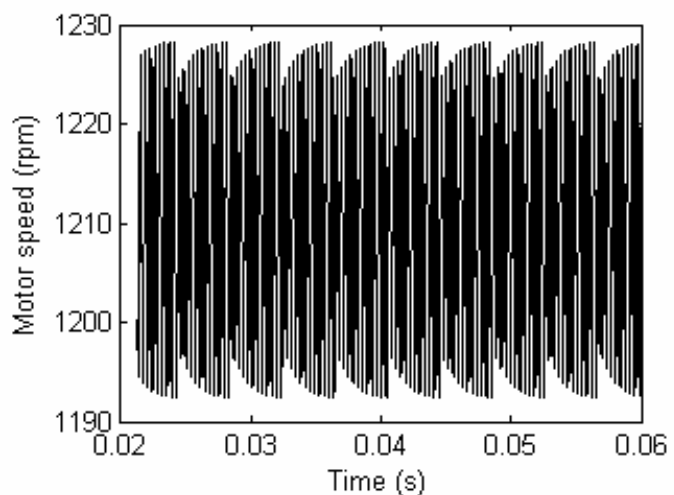

(a)

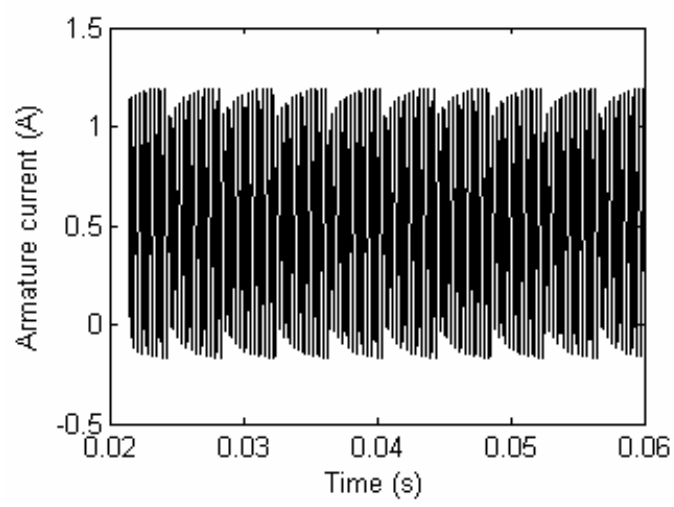

(b)

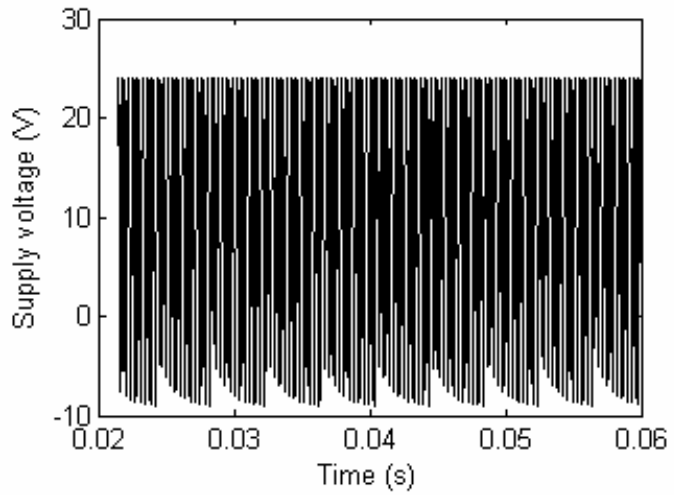

(c)

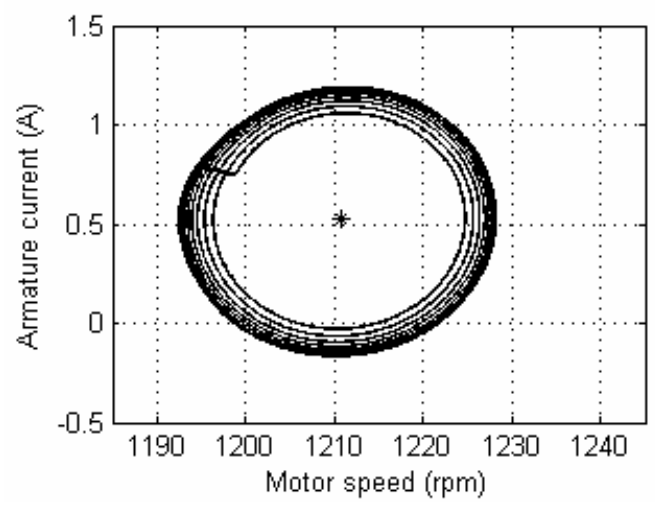

(d)

Figure 5. The equilibrium locates at a non-zero point with a large $\delta$. (a) Motor speed. (b) Armature current. (c) Supply voltage. (d) Phase portrait.

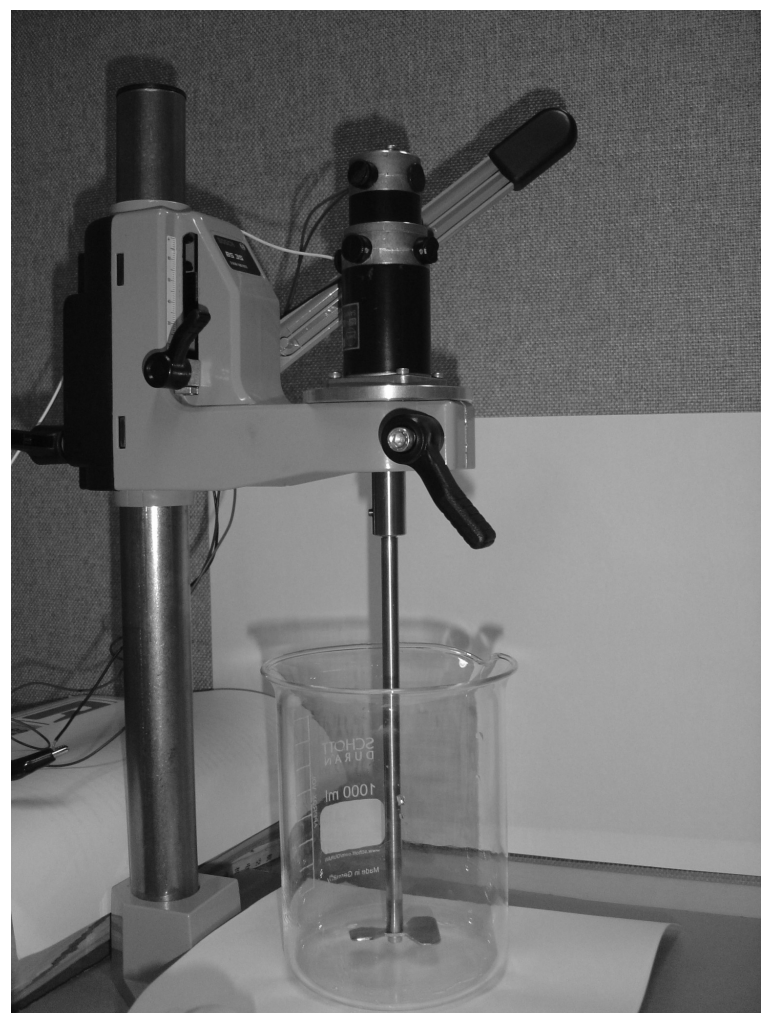

Figure 6. Experimental set-up.

\section{REFERENCES}

[1] D.J. Lamberto, F.J. Muzzio, P.D. Swanson and A.L. Tonkovich, "Using time-dependent rpm to enhance mixing in stirred vessels," Chemical Engineering Science, vol. 51, 1996, pp. 733-741.

[2] N. Harnby, M.F. Edwards and A.W. Nienow, Mixing in the Process Industries, 2nd ed., Oxford: Butterworth-Heinemann, 1992, pp. 1-2.

[3] F. Raynal and J.N. Gence, "Energy saving in chaotic laminar mixing," Journal of Heat and Mass Transfer, vol. 40, 1997, pp. 3267-3273.

[4] M.M. Alvarez-Hernández, T. Shinbrot, J. Zalc and F.J. Muzzio, "Practical chaotic mixing," Chemical Engineering Science, vol. 57, 2002, pp. 3749-3753.

[5] J.H. Chen, K.T. Chau and C.C. Chan, "Analysis of chaos in currentmode controlled dc drive systems," IEEE Transactions on Industrial Electronics, vol. 47, 2000, pp. 67-76.

[6] Y. Gao and K.T. Chau, "Design of permanent magnets to avoid chaos in PM synchronous machines," IEEE Transactions on Magnetics, vol. 39, 2003, pp. 2995-2997.

[7] J.H. Chen, K.T. Chau, S.M. Siu and C.C. Chan, "Experimental stabilization of chaos in a voltage-mode DC drive system," IEEE Transactions on Circuits and Systems I: Fundamental Theory and Applications, vol. 47, 2000, pp. 1093-1095.

[8] K.T. Chau, S. Ye, Y. Gao and J.H. Chen, "Application of chaoticmotion motors to industrial mixing processes," IEEE Industry Applications Society Annual Meeting, vol. 3, 2004, pp. 1874-1880.

[9] T. Kousaka, T. Ueta and H. Kawakami, "A method for generating a chaotic attractor by destabilization," Electronics and Communications in Japan, vol. 80, 1997, pp. 73-81.

[10] P. Mavros, "Flow visualization in stirred vessels, a review of experimental techniques," Transactions of the Institution of Chemical Engineers, Part A, vol. 79, 2001, pp.113-127.

[11] G. Ascanio, M. Brito-Bazán, E. Brito-De La Fuente, P.J. Carreau and P.A. Tanguy, "Unconventional configuration studies to improve mixing times in stirred tanks," The Canadian Journal of Chemical Engineering, vol. 80,2002 , pp. 558-565. 


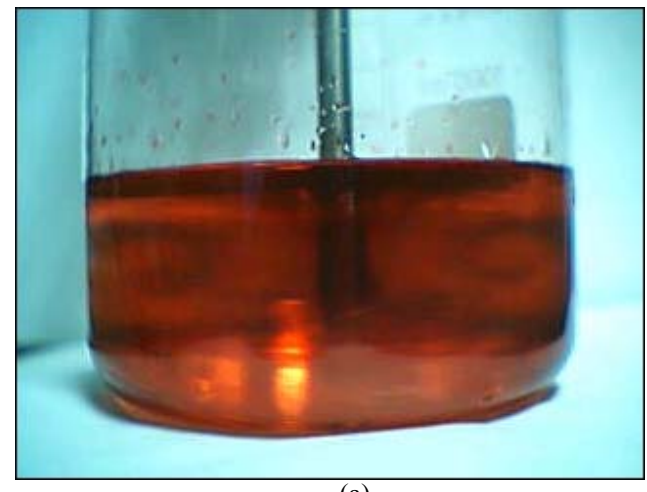

(a)

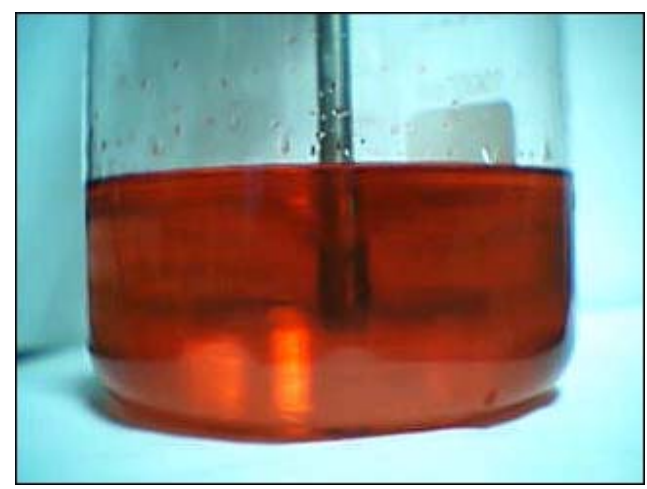

(b)

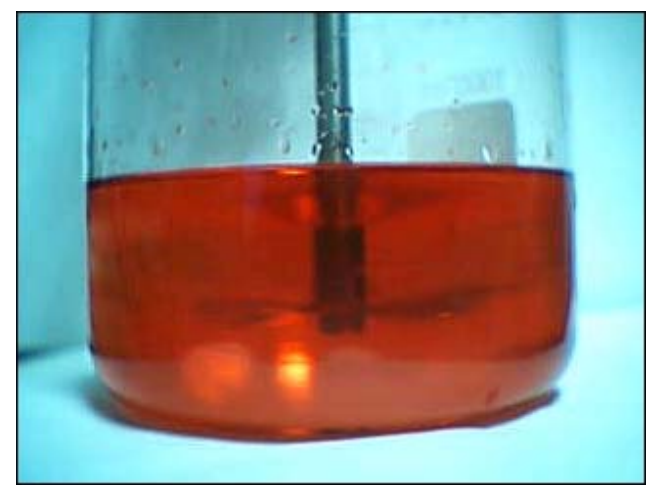

(c)

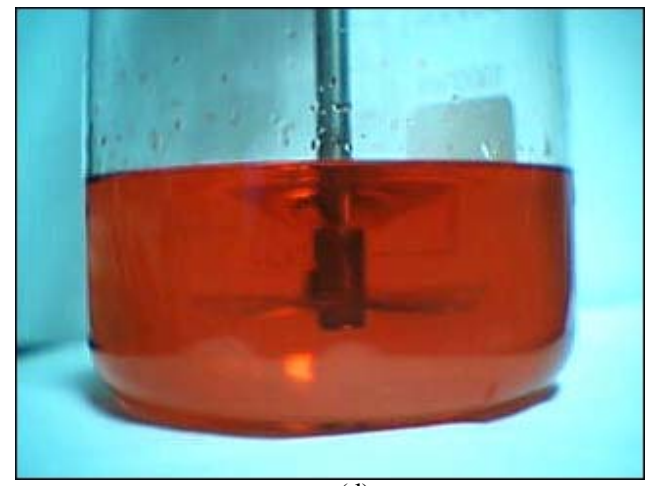

(d)

Figure 7. Coloration during chaotic mixing. (a) After 9 s. (b) After 14 s. (c) After 20 s. (d) After $60 \mathrm{~s}$.

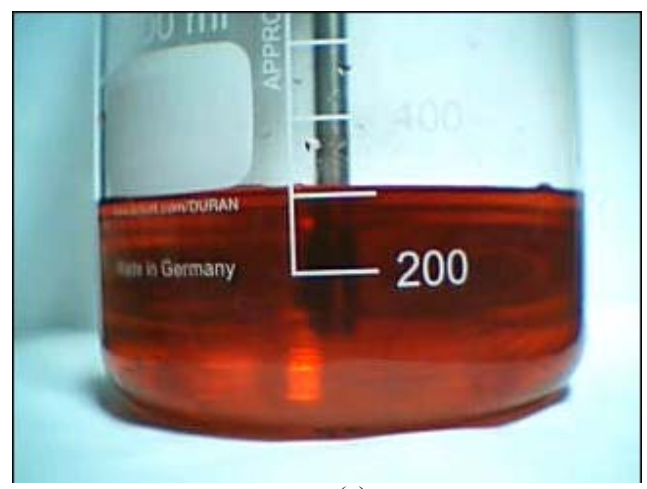

(a)

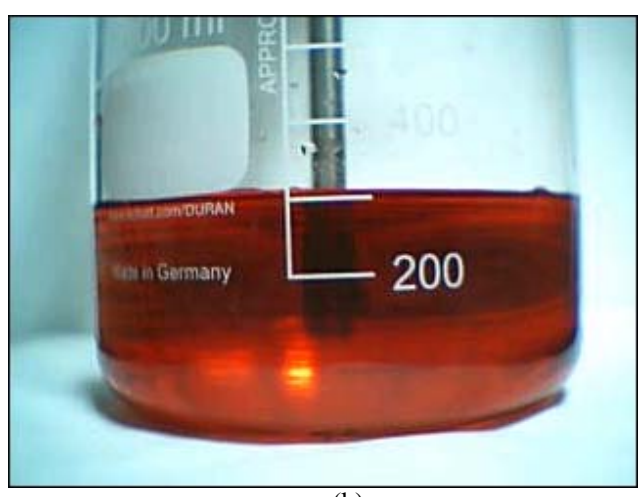

(b)

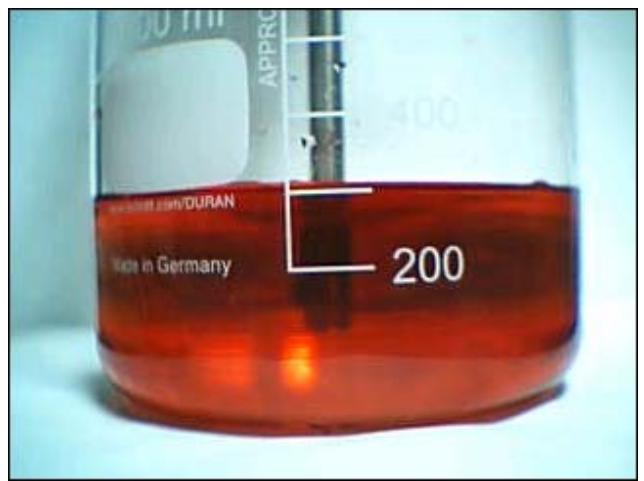

(c)

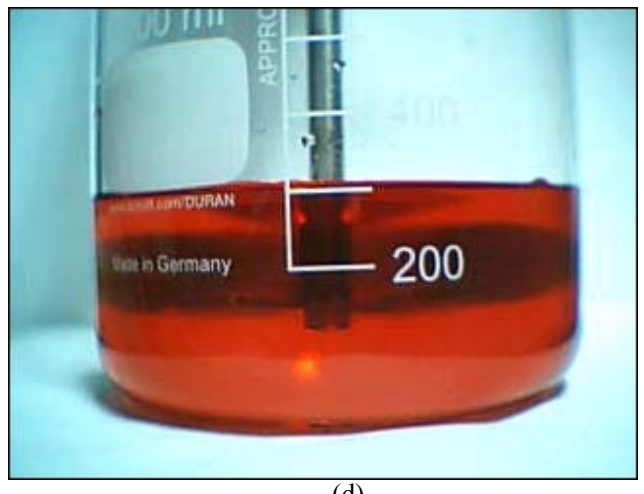

(d)

Figure 8. Coloration during normal constant speed mixing. (a) After 9 s. (b) After 14 s. (c) After 20 s. (d) After 300 s. 